\title{
RF Energy Harvesting Wireless Networks: Challenges, and Opportunities
}

\author{
Amar Esse Ahmed', Khaizuran Abdullah ${ }^{2}$, Mohamed Hadi Habaebi ${ }^{3}$, Huda Adibah Mohd. Ramli, \\ Ani Liza Asnawi ${ }^{5}$ \\ 1,2,3,4,5 Department of Electrical and Computer Engineering, Kulliyyah of Engineering, International Islamic University, \\ Gombak Road, 53100, Selangor, Malaysia
}

\begin{tabular}{l} 
Article Info \\
\hline Article history: \\
Received Feb 3, 2020 \\
Revised Feb 23, 2021 \\
Accepted Mar 3, 2021 \\
\hline Keywords: \\
RF Energy \\
Energy Harvesting \\
5G \\
SWIPT \\
Power management \\
Power Splitting \\
\hline
\end{tabular}

\begin{abstract}
Energy harvesting wireless networks is one of the most researched topics in this decade, both in industry and academia, as it can offer self-sustaining sensor networks. With RF energy harvesting (RF-EH) embedded, the sensors can operate for extended periods by harvesting energy from the environment or by receiving it as an Energy signal from a hybrid base station (HBS). Thus, providing sustainable solutions for managing massive numbers of sensor nodes. However, the biggest hurdle of RF energy is the low energy density due to spreading loss. This paper investigates the RF-EH node hardware and design essentials, performance matrices of RF-EH. Power management in energy harvesting nodes is discussed. Furthermore, an information criticality algorithm is proposed for critical and hazardous use cases. Finally, some of the RF-EH applications and the opportunities of 5G technologies for the RF-EH are introduced.
\end{abstract}

Copyright $(\odot) 2021$ Institute of Advanced Engineering and Science. All rights reserved.

\section{Corresponding Author:}

Khaizuran Abdullah,

Department of Electrical and Computer Engineering,

Kulliyyah of Engineering, International Islamic University Malaysia,

Gombak Road, 53100, Selangor, Malaysia.

Email: khaizuran@iium.edu.my

\section{INTRODUCTION}

The spread of wireless networks and the increasing number of wireless-enabled nodes exacerbated the burden on the power grid by dragging more energy from the grid[1]. This expansion also implies that more energy is sent through the air than ever before. According to the well-established first law of thermodynamics of energy conservation, energy cannot be destroyed or created. It can only be transferred from one form to another. The energy harvesting notion in wireless communication refers to the process of collecting and storing the RF energy from the environment in order to achieve self-sustaining nodes [2] that can operate for extended lifetimes without the need to recharge in conventional ways [3].

The energy harvesting field has gone through enormous amounts of research, leading to the emerging of energy harnessing systems that can harvest ambient energy from the environment. Energy harvesting wireless nodes are capable of harvesting and storing RF energy from the surroundings [4]. This RF energy could be ambient or interference or, in other words, a signal not intended for the harvesting node; hence, ambient RF energy can be categorized as a green communication [5], [6]. When a dedicated transmitter is used to send energy to a harvesting node, this type of communication could be more categorized as a convenience rather than green because of the excessive energy loss in the wireless media due to fading [7].

The development in the wireless field and the processing power lead to the emergence of IoT (internet of things), where small wireless devices are used to collect data like sensor networks or automate everyday things like refrigerators[8]. In wireless sensor networks (WSN), nodes are required to stay on standby for years [9]. However, due to the current limitations in battery technology, it is challenging to design a battery that can stay for that long without compromising the efficiency of the intended application[10]. 
Equipping sensor network nodes with energy harvesting systems is essential to stay online for longer durations. One of the main challenges in modelling and designing an energy harvesting communication system is energy management [11]. Due to the randomness of the ambient energy in general, the available RF ambient energy at any specific point of time is random. Therefore, it is necessary to design optimal transmission policies to model the time-varying ambient energy so that the system can effectively exploit the arriving power. On the other hand, when using a dedicated RF transmitter, the available energy density at the receiver input is very low due to high spreading loss. Thus, having restricted power management algorithms for the RF energy harvesting (RF-EH) system is necessary [12].

Traditional wireless systems only extract information from the received signal [13]. Wireless energy harvester extracts both information and power from the received signal. Accordingly, Simultaneous wireless information and wireless transfer (SWIPT) [14] was introduced, where the information and energy are extracted simultaneously from the received signal. SWIPT can be classified into two categories.

The first category assumes that the receiver can simultaneously harvest and extract information from the received signal[15] using the same circuit. However, this category's feasibility is in question, as there is no practical hardware implementation that can extract information and energy at the same time[16]. In the second category, which is the more practical one, the receiver performs power splitting (PS) using different circuitry for energy and information, where it splits the received signal between the two circuitries.

One implementation of EH networks, which have emerged recently, is Cooperative communications. These systems utilize the geographical distance between network nodes, using nodes that are near the access station as relay portals for the remote nodes. Thus, reducing energy loss substantially by a unit of distance. Energy and information scheduling methods in cooperative relay communications have been well studied to achieve the best trade-off between energy harvesting and spectrum efficiency in [17]

The rest of this paper is organized as follows: Section 2 discusses some of the most popular harvestable energy sources.

Section 3 RF-EH node hardware. Section 4 Co-located wireless information and power transceiver architecture. Section 5 Power management in RF-EH WSN. Section 6 RF-EH limited energy Challenges and Solutions. Section 7 RF-EH applications and opportunities. And finally, section 8 concludes the article.

\section{ENERGY SOURCES}

In wireless energy harvesting networks, there are different sources of energy available for harnessing depending on the behaviour and the nature of these sources, and they can be categorized into the following:

1: Predictable and controllable: in this kind of energy source, the energy origin is under the network's control. A good example can be a wireless energy transmitter, where the energy is delivered depending on the demand of the sensing node.

2: Predictable but uncontrollable: these are ambient energy that can be predicted with a reasonable accuracy using simple mathematical models. However, it cannot be generated or controlled based on the network requirements - for instance, solar energy.

3: Unpredictable and uncontrollable: This energy source does not follow any pattern, and it is completely random. Thus, it cannot be controlled to generate the required energy by the network ondemand; one example of such energy is mechanical vibration using a piezoelectric generator [18].

\subsection{Light Energy}

Light is one of the most efficient energy sources to harvest. Light photons can be transformed into electricity through photovoltaic cells. For outdoor scenarios, solar energy may represent a reliable source of power for autonomous sensor nodes. Solar power has been used in many applications to replace batteries and power from the grid. The solar panel converts light radiation from the sun to electricity. This electricity is used to operate different types of wireless networks. Solar harvesting systems have high efficiency and relatively low cost. Furthermore, the energy level provided by the sun is relatively high. For instance, the energy level just outside the atmosphere is $1361 \mathrm{w} / \mathrm{m}^{2}$ [19] although this number reduces to less than half when it reaches the earth's surface due to atmospheric attenuation, solar energy is still considered a high power source compared to other wireless ambient sources. The downside of solar energy is its reliance on the earth's movement, making it available for less than 12 hours in most earth regions. When the sun disappears, the solar Reliant wireless networks had to rely on a battery or another energy source to mitigate outage. Likewise, solar energy is not available in indoor scenarios. Mostly, solar energy may be uncontrollable; however, it can be predicted [20], and usually, it provides superior power compared to other existing energy sources.

Recently an infrared transmitter for wireless energy harvesting systems was introduced in [21]; this transmitter can deliver $2.25 \mathrm{~W}$ to a device at a distance of $5 \mathrm{~m}$ as show In Figure 1. 


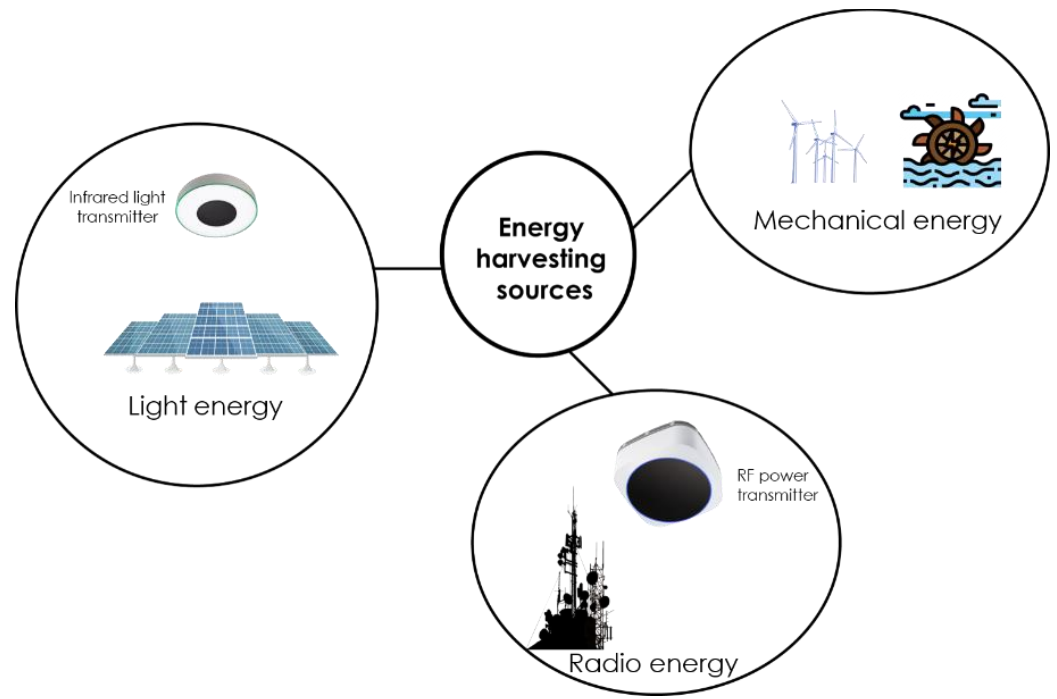

Figure 1. Harvestable energy sources

\subsection{Mechanical energy}

Mechanical energy from the natural motion of air, water, vibration from a pedestrian walking across the sidewalk or the moving cars across a bridge can be harvested and transformed into electricity. The mechanical energy sources vary in their effectiveness and randomness. From least effective like the vibration to the most reliable and effective hydropower. Some countries like Norway produces almost all of their energy from hydropower, and globally, hydropower accounts for $90 \%$ of the renewable energy [22]. In Malaysia, the total hydropower production in 2016 Was $17.93 \mathrm{TWh}$, which represents $11 \%$ of countries electricity generation [23].

Vibration usually uses piezoelectric material that generates electricity when stress is applied to its piezoelectric material [24]. The output of a piezoelectrical transducer is AC. Thus, a rectifying circuit that converts the current into DC is required in order to store the energy, as shown in Figure 2.

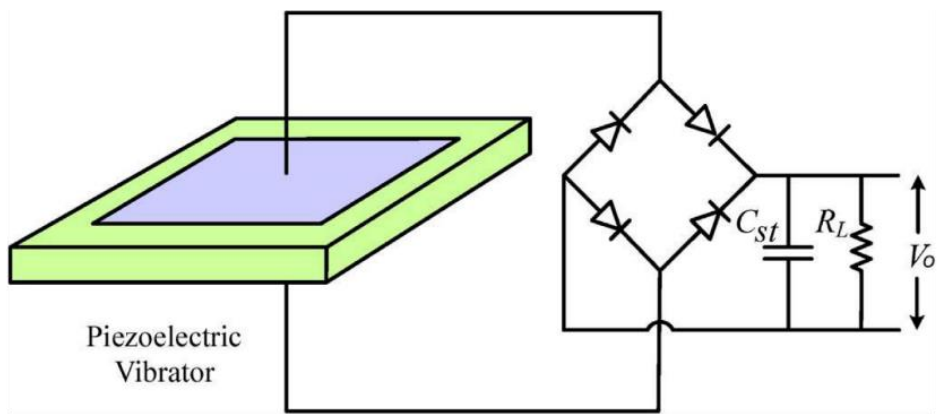

Figure 2. Piezoelectric transducer connected to a rectifying circuit [25].

\subsection{Radio Energy}

$\mathrm{RF}$ energy is the energy carried by the radio spectrum, ranging from $30 \mathrm{kHz}$ to $300 \mathrm{GHz}$, as illustrated in Figure 3. RF energy is available both outdoors and indoors, day and night, making it an appealing choice for low power sensor devices. The source of RF energy could be a dedicated RF transmitter or ambient. In an ambient EH scenario, the harvester is harnessing energy from random RF signals from other users in the environment, like TV signals, WIFI and cellular stations. On the other hand, when implementing a dedicated RF transmitter, the harvestable power can be controlled, yielding higher reliability, especially in WSN where a continuous stream of a power supply is required, allowing sensor nodes to operate in a semiautonomous mode in terms of power. In addition, due to the remarkably confined size of the RF-EH circuit [26], it can be easily embedded into small devices, unlike traditional $\mathrm{EH}$ techniques, which sometimes involve large mechanical parts like wind turbines. Thus, RF-EH provides the ability to design compact and low-cost devices. 
For a long time, wireless was limited for information transmission to the extent that there is' communication' beside it whenever the 'wireless' word is stated. Until Harry stockman published his paper [27] in 1948 named "Communication by Means of Reflected Power". The Stockman work was considered as the first prototype of an RF identification (RF-ID). In 1975 Koelle presented an RF-ID system [28]. This system consisted of a continuous wave RF reader with a $1 \mathrm{Ghz}$ transmitter and an RF tag. The RF tag used the signal to change the load in its rectifier and use it for reradiating using a $20 \mathrm{KHz}$ subcarrier. As the RF tag rectified some portion of the signal and use it to transmit back, it can be considered as the first wireless powered communication system.

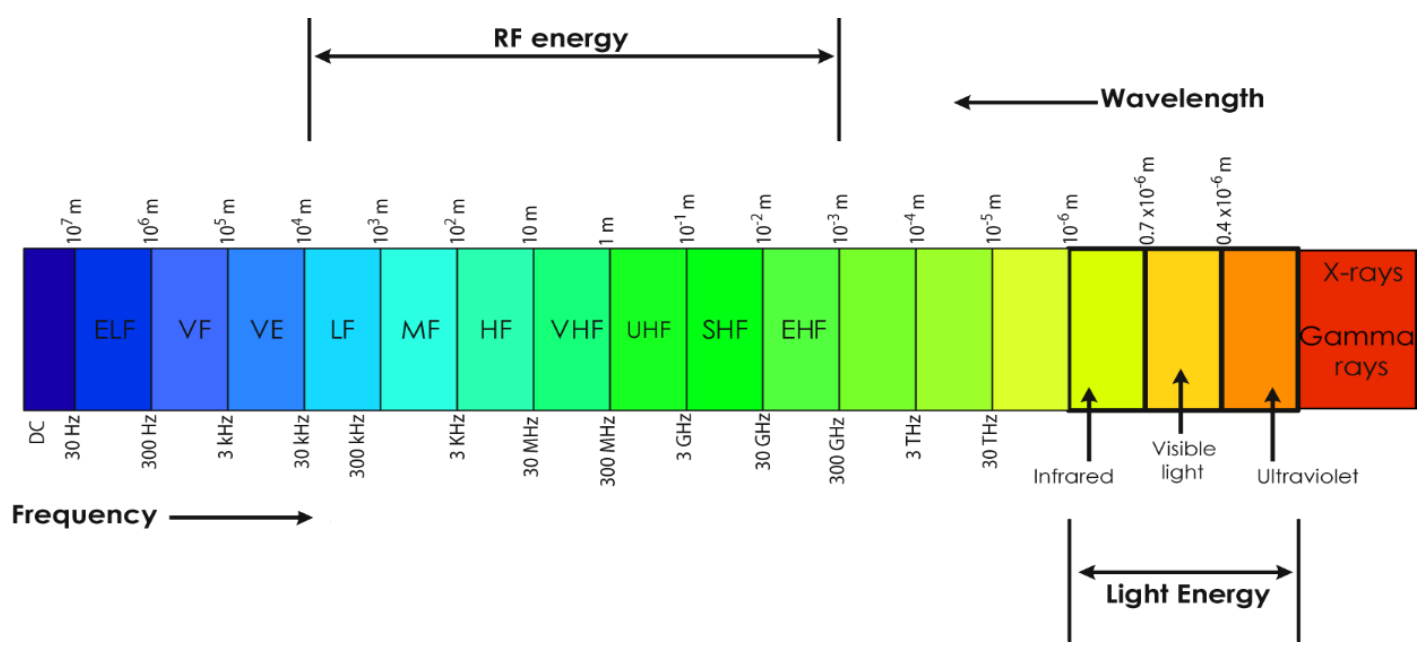

Figure 3. Electromagnetic wave spectrum

\section{RF-EH NODE HARDWARE}

A sensor node with EH capability must have circuitry that can handle both IP and EH. In this section of the survey, we will briefly look at the essential parts of the EH hardware.

\subsection{Design Essentials of RF EH circuit}

RF EH circuitry requires three necessary components to be able to harvest energy; as illustrated in Figure 4, they are a matching circuit, voltage multiplier, and energy storage.

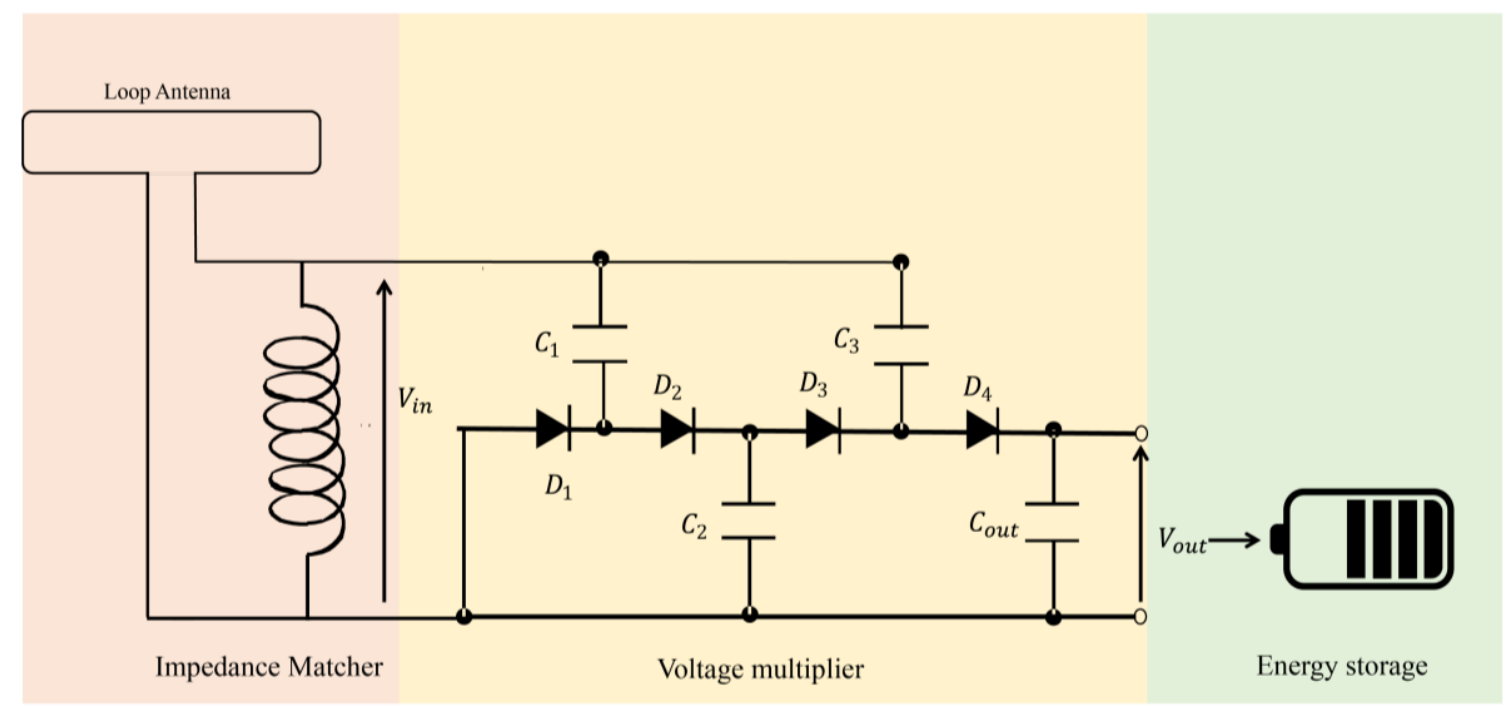

Figure 4. RF energy Harvester primary circuit

\subsubsection{Matching Circuit}

To ensure the maximum conversion ratio, the impedance of the $\mathrm{EH}$ circuit and the antenna should be the same in order to prevent the received RF from reflecting back to the antenna and then to the air. The 
matching circuit should be specifically designed for the intended power and frequency to yield the highest conversion efficiency [29].

\subsubsection{Rectifier and Voltage Multiplier}

Knowing the alternating nature of the RF signal, the Received RF energy from the antenna must be converted to a Direct current (DC) in order to store it in an energy storage unit such as a supercapacitor or a battery.

However, due to the low DC output, the input voltage must be elevated to a practical level to power an integrated circuit [30]. There are different voltage uplifting schemes in the literature, such as inductor-based multipliers, Cockcroft Walton and Dickson multipliers. Furthermore, some literature [2] uses MOSFET transistor technology to overcome the relatively high threshold voltage of diode-based multipliers. However, this method requires to be implemented using complementary metal-oxide-semiconductor (CMOS). Such CMOS implementations are not easily accessible and cannot be adopted at a board-level application [31]. Inductor based potential lifters are expensive and bulky in terms of size[32], making them impractical in integrated applications like sensor nodes. Cockcroft-Walton and Dickson schemes are generally suitable for board-level low energy applications, shown in Figure 5 and Figure 6, respectively.

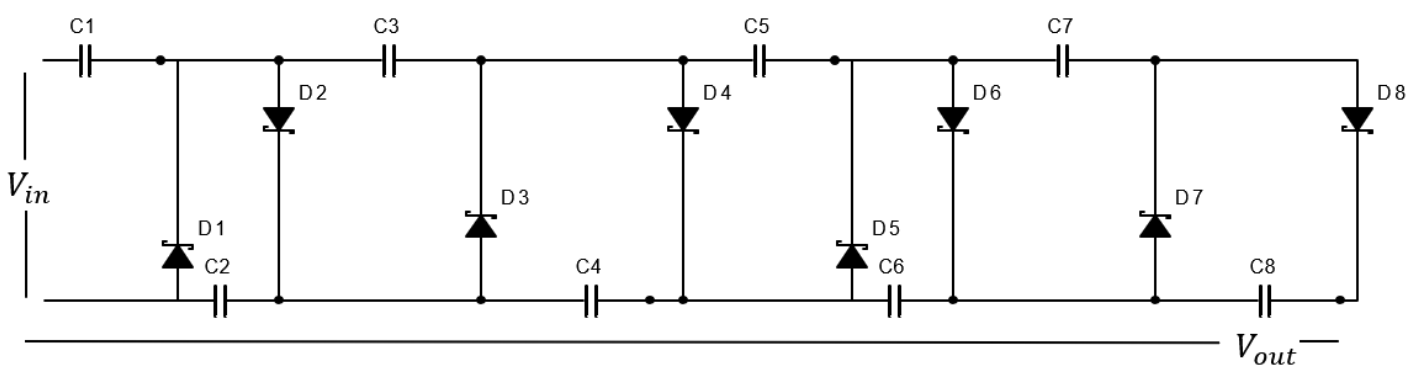

Figure 5. Cockcroft-Walton multiplier

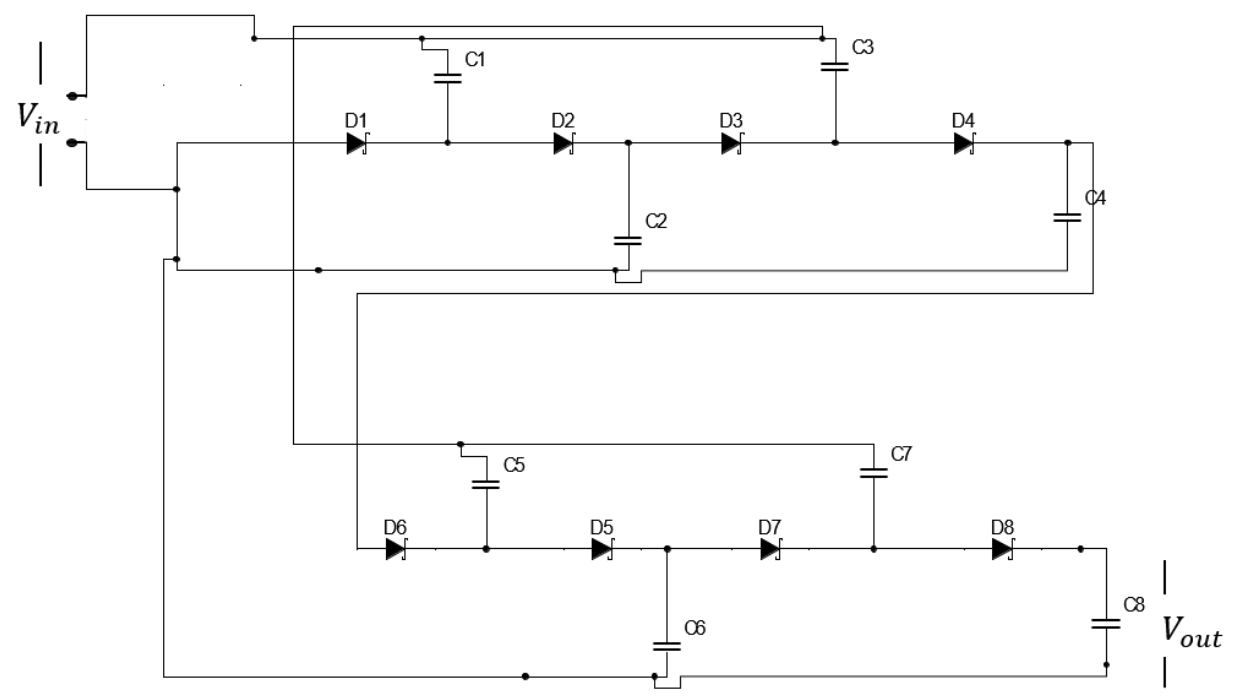

Figure 6. Dickson multiplier

Due to the low RF power intensity at the receiver, selecting an appropriate diode is of paramount importance for the system to operate at that level of weak RF input. In [29], an implementation of an RF harvesting sensor system is presented using Schottky diodes, which can operate at a forward voltage of $0.15 \mathrm{~V}$.

Schottky diodes can operate at very high frequency, with low power input. For example, Skyworks rectifier series SMS7621 can operate at frequencies up to $24 \mathrm{GHz}$ [33]. Diode-based Voltage multiplier arranges diodes in cascaded stages to increase the voltage output, depending on the application and the required voltage. In theory, the number of stages is directly proportional to the output voltage. However, practical considerations such as parasitic capacitance bound the number of admissible stages [34]. In the CockcroftWalton arrangement, due to the series cascade, as shown in figure 5, the total diode impedance is very high. Compared to the parallel scheme in Dickson, Figure 6, where every diode in the cascade has a connection to the RF source. An important consideration when designing a Dickson charge multiplier is the last capacitor 
tolerance as the voltage difference between its plates is the output of the charge multiplier. A solution for the high voltage tolerance requirements of the Dickson output capacitor is presented in [35], where Dickson and Cockcroft-Walton architects are combined.

\subsection{Performance metrics when designing RF-EH circuit}

- Energy sensitivity: is the minimum energy that the RF-EH circuit can sense and convert to DC. The sensitivity of an RF-harvester should be high in order to harvest the low-density RF power from the environment.

- Conversion efficiency: is the ability of the harvester to convert the incident energy at the antenna to DC power; in other words, the ratio of received RF energy to the DC output from the multiplier. Conversion efficiency depends on the RF field strength and the harvester hardware properties[36].

\section{CO-LOCATED WIRELESS INFORMATION AND POWER TRANSCEIVER} ARCHITECTURE

This section elaborates the receiver architecture for single-input receivers where the information decoder and energy harvester share the same antenna, implying the channel characteristics for the information and energy signal will be the same. The switching architecture can be classified into time switching (TS) and power switching (PS), as illustrated in Figure 7.

\subsection{Time switching mode}

In TS scheduling mode, the total time of transmission is divided between Energy Harvesting (EH) and information processing (IP) $T$ for information transmission and $(T-1)$ for energy. Thus, resulting in a Halfduplex communication system [37], as shown in Figure 7(A). The harvested power at the transmitter can be modelled mathematically as $Q^{T s}=\xi(T-\tau) p_{t} h$.where $Q^{T s}$ is the harvested energy; $\xi$ is the efficiency of the energy harvester conversion, $T$ is the total time, $\tau$ is the time elapsed for transmitting the information signal, $h$ represents channel gain, and $p_{t}$ is the transmitted power from the transmitter.

One of the simplest RF-EH TS systems is a point to point EH. In this form of EH, the transmitter or the base station first sends energy to the receiver node; then, the receiver uses the received energy to send information back to the base station, as shown in Figure 8.

The introduction of EH entails new design challenges at the destination node. Specifically, the EH node can only transmit no more than what it already harvested in terms of power allocation. In other words, the amount of information it can transmit at any given time strictly flows the amount of harvested energy, which is contrary to the conventional wireless systems where the available energy is unbounded, for instance, base station or mobile phones where massive amounts of stored energy are available from lithium-ion batteries.

To model a simple point to point EH system, let us assume a system where the destination node (DN) depends solely on the harvested energy. The DN first harvests and stores the received energy and then starts to transmit back to the hybrid base station (HBS). The system can be visualized as a WSN where the sensor nodes send the collected data to the HBS at a time interval equivalent to the frequency of the received energy. All the system nodes are assumed to have a single antenna. Assuming the DN first performs EH and then starts transmitting, the system is half-duplex. Thus, each power transfer and information transmission share a time interval of T seconds. So, the DN has to harvest energy at a frequency of $K$ time intervals at a total time of $K T$ seconds in order to have enough energy for data transmission.

From the above assumptions, we will have a two-stage communication procedure. In the first stage, the HBS transfers energy to the DN, which is expressed as follows.

$$
y_{k}=h_{k} \sqrt{P_{s}} S_{k}+n_{k}
$$

Where $y_{k}$ is the energy harvested at $k$ interval, $h_{k}$ is the channel coefficient and $s_{k}$ is the energy signal, and $n_{k}$ is the additive noise. In the second stage, the DN transmits data back to the HBS using the harvested energy.

$$
r=g \sqrt{P_{x}} x+n
$$

Where $\boldsymbol{x}$ is the data signal, $g$ is the channel fading coefficient and $\sqrt{P_{x}}$ is the power used for transmission. 

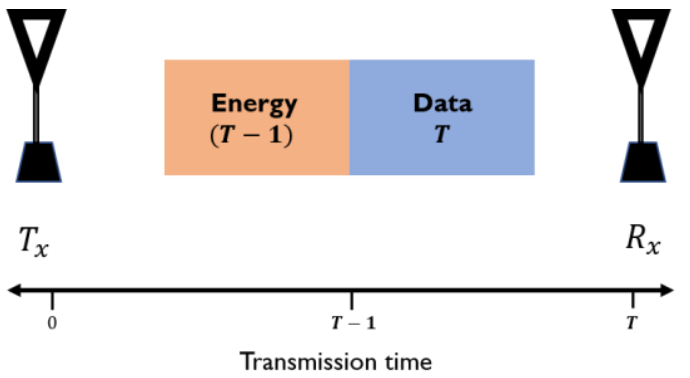

(a)
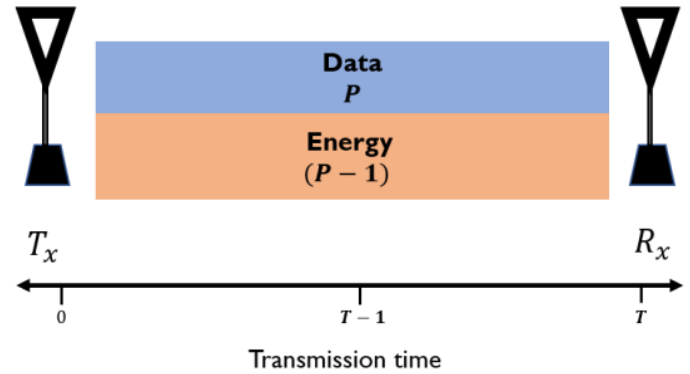

(b)

Figure 7. (a) Time switching mode, (b) Power switching mode

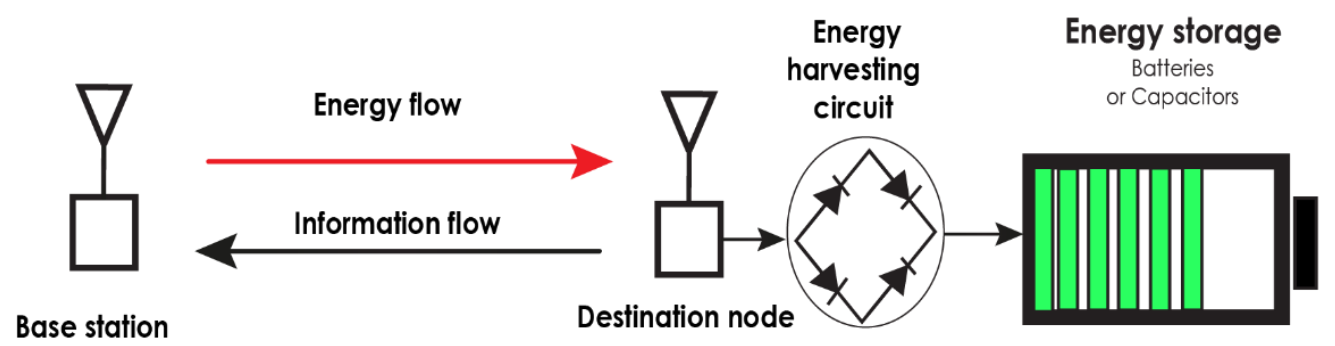

Figure 8 Point to Point Energy Harvesting system

\subsection{Power Splitting Mode}

In power splitting, the received signal is divided between the energy harvester and the information decoder. Theoretically, PS mode is full-duplex as it transmits energy and information signal simultaneously, as shown in Figure 7(b).

\section{POWER MANAGEMENT IN RF-EH WSN}

\subsection{Limited energy}

Considering THE RF signal density at the receiver side, it is critical to utilize the harvested energy in the most efficient way possible. Different power management methods have been proposed in the literature over the years. Energy signals arriving at the sensor node are modelled as packets to simplify the harvesting process description. These discrete energy packets could vary in size and arrive at random time points depending on the energy source. In Offline power management methods, the receiver knows the statistical distribution of arriving power in advance. Thus, the aggregated harvested energy can be illustrated as a staircase curve, as shown in Figure 9. The staircase representation comes from the limited battery capacity of the harvesting node $D_{1}$ And the energy harvest constraint $D_{2}$. The aggregated energy consumption must stay in the area between $D_{1}$ and $D_{2}$ which can be described as a tunnel. If the consumption curve is beneath $D_{2}$ Battery overflow will occur. While, on the other hand, if the curve exceeds $S_{2}$ The battery can no longer be able to provide sufficient energy. Thus, the consumption curve $M$ that maximizes data rate under constrained energy resources should stay in the feasibility tunnel; this can be formulated as follows.

$$
\begin{array}{cc}
M 1: \arg \max \int_{0}^{t_{i}} r(t) d t & \\
\text { s.t } \quad S_{1}: \int_{0}^{t_{i}} p(t) d t \leq \sum_{j=0}^{t_{j} \leq t_{i}} e_{j} & i=1, \ldots \ldots, N+1, \\
S_{2}: \sum_{j=0}^{t_{j} \leq t_{i}} e_{j}-\int_{0}^{t_{i}} p(t) d t \leq e_{\max } & i=1, \ldots, N,
\end{array}
$$




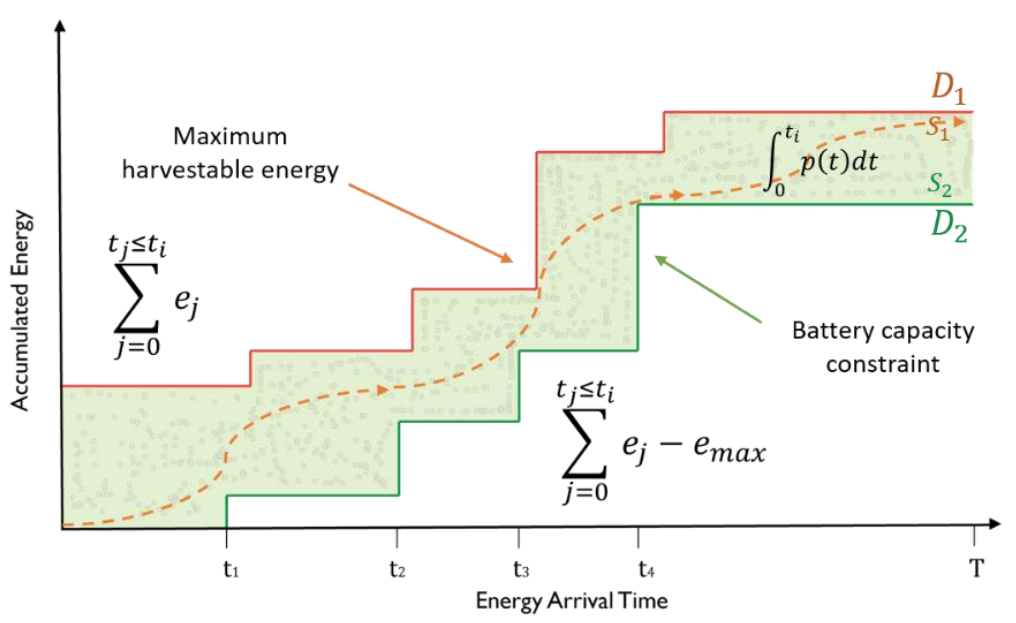

Figure 9. Power management tunnel recreated from [38]

Where $r(t) d t$ is the data rate and $t_{i}$ and $e_{j}$ are energy packet arrival time and amount of energy in each packet, respectively. $e_{\max }$ is the maximum storable energy due to battery size constraints. $S_{1}$ and $S_{2}$ are constraints representing the maximum harvestable energy and battery capacity respectively, $p(t)$ is the instantaneous power transmission at any given time $t_{i}$.

\subsection{Limited buffer capacity}

The optimal transmission power should stay the same between energy harvests [39] to maximize the throughput for the RF-EH sensor node. This simplifies the power management problem into a piecewise optimization problem. The data buffer capacity is pawned due to the size and cost of the sensor node; thus, the accumulated information may overflow from the data storage unit if not discharged timely by sending out the data.

THE optimal energy harvesting strategy is aware of the limited data buffer by vacating data from the buffer timely, using the lowest energy cost (energy per bit). In practical WSN, a dedicated energy transmitter is required to transfer energy and charge the sensor network. In [40], a dual tunnel strategy to solve the optimal energy request strategy is presented. The strategy calculates the minimum energy required by a sensor node to transmit the accumulated data. The calculation takes into consideration the limited data storage. This can be formulated as follows.

$$
\begin{array}{cc}
M 2: \arg \min \int_{0}^{t_{i}} p(t) d t, & \\
\text { s.t } \quad S_{1}: \int_{0}^{t_{i}} r(t) d t \leq \sum_{j=1}^{t_{j} \leq t_{i}} D_{j} & i=1, \ldots \ldots, N+1, \\
S_{2}: \sum_{j=0}^{t_{j} \leq t_{i}} D_{j}-\int_{0}^{t_{i}} p(t) d t \leq D_{\max } & i=1, \ldots, N,
\end{array}
$$

$D_{\max }$ stands for the maximum capacity of the data buffer. Where $r(t)$ is the transmission rate; $t_{i}$ and $D_{j}$ are the arrival time and size of the packet $i$, respectively. The transmission power and rate are related through power rate function $f(r(t))=p(t) . S_{1}$ and $S_{2}$ are data arrival and maximum buffer capacity constraints, respectively. The optimization problem $M 2$ minimizes the energy consumed by the sensor node while transmitting all the data packets in the limited data buffer. As illustrated in Figure 10, a realistic data tunnel is based on the data arriving at time $t$ and the limited buffer capacity. 


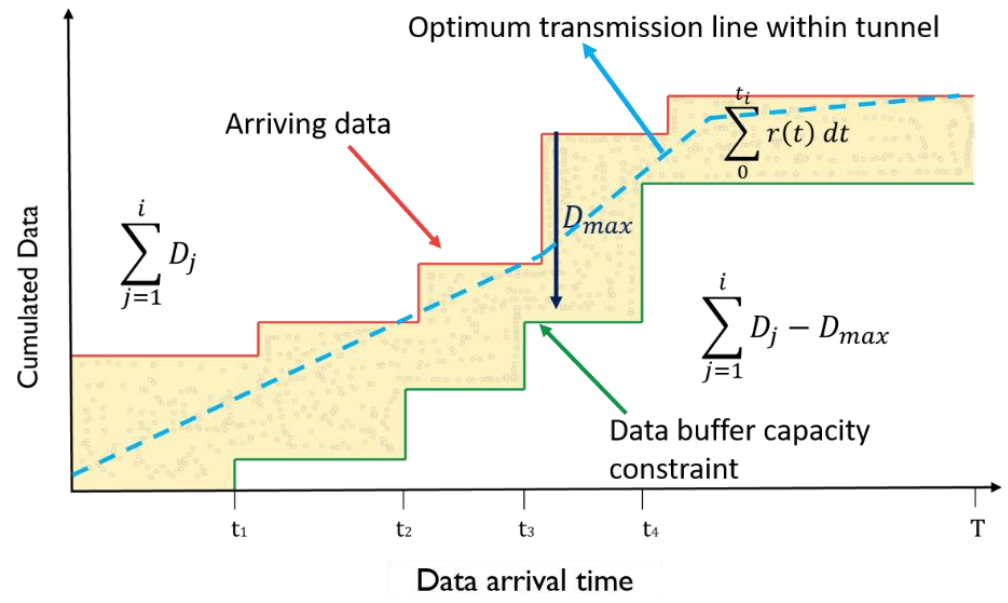

Figure 10. Data management tunnel recreated from [40]

\section{RF-EH LIMITED ENERGY CHALLENGES AND SOLUTIONS}

Adopting a system with WIPT capability usually involves providing a dedicated RF energy transmitter to avoid uncertainties in ambient RF energy. Due to spreading loss, the energy signal will diminish multiple folds with unit distance, especially at high frequencies, making it hard to establish wide-area WIPT systems. Most of the research in the RF-EH field does not address this problem and conduct research in a closed area where the transmitter and receiver are closely placed. In Figure 11 and Table 1, some of the FCC auctioned 5G spectrum[41] free-space path loss (FSPL) are listed. The power levels in the best-case scenarios are in $n W$ scale, which is very low. Even in relatively lower frequencies $0.3-3 \mathrm{GHz}$ the energy levels are in the same category [42] for ambient energy from different RF sources, as illustrated in Figure 12. Due to the maximum RF radiation regulations, the amount of transmittable power is limited [43], thus reducing the options of powering WSN located in the far-field range.

Table 1. The FSPL for some of the FCC auctioned frequencies for 5G

\begin{tabular}{lllll}
\hline Frequency $\mathrm{GHz}$ & FSPL $100 \mathrm{~m} \mathrm{~dB}$ & FSPL $1 \mathrm{~km} \mathrm{~dB}$ & FSPL $5 \mathrm{~km} \mathrm{~dB}$ & FSPL $10 \mathrm{~km} \mathrm{~dB}$ \\
\hline 3.4 & 83 & 103 & 117 & 123 \\
24 & 100 & 120 & 134 & 140 \\
28 & 101 & 121 & 135 & 141 \\
39 & 104 & 124 & 138 & 144 \\
47 & 105 & 125 & 139 & 145 \\
\hline
\end{tabular}
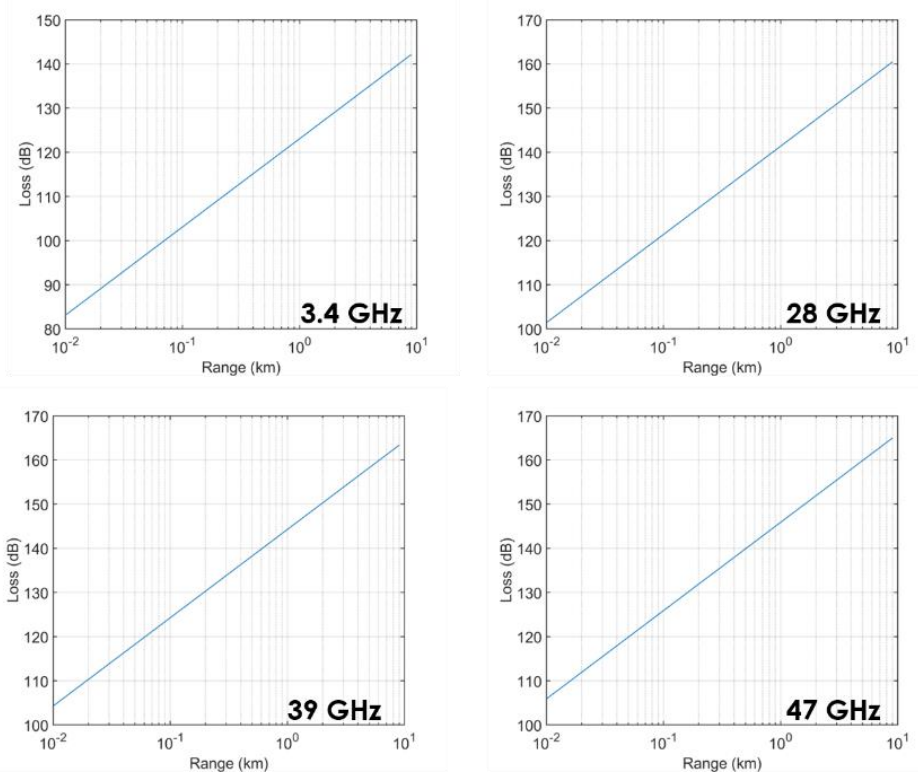

Figure 11. The FSPL for some of the FCC auctioned frequencies for $5 \mathrm{G}$ 


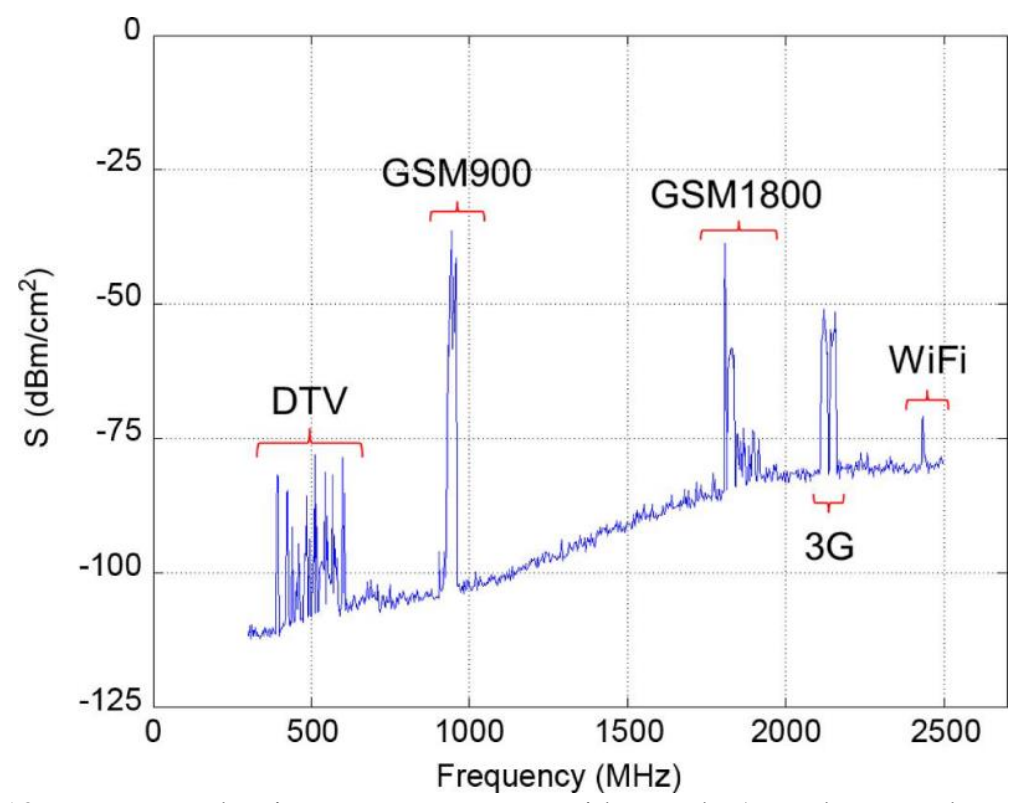

Figure 12. RF power density measurements outside London's Underground station [42]

The uncertainty in the arriving energy introduces challenges in designing transmission policies, especially when harvesting from the ambient RF. For example, when the sensor node wants to send information, which by the way, arrives randomly since the sensor does not know the information it collects in advance, the available energy at that specific point of time may not be sufficient for transmission, or the sensor may run out of energy before the transmission finishes. Thus, it is necessary to adopt transmission policies that consider the energy constraint and the significance of the information in the buffer. Consequently, we propose two basic algorithms for information transmission management.

Algorithm one: this algorithm takes into consideration the importance and criticality of the collected information. Such an approach could be of paramount importance in hazardous environments where immediate action is a must, depending on the collected data. The information with the highest entropy will be assigned the lowest characters or bits, and thus, it will require less energy, and consequently, the percentage of transmission failure due to insufficient energy will be very low for critical information. Brief exposure to this algorithm is shown in Table 2.

Table 2. Transmission management algorithm one

\begin{tabular}{llll}
\hline criticality & Character & $\begin{array}{l}\text { Energy per character/Total } \\
\text { energy capacity } \%\end{array}$ & $\begin{array}{l}\text { Required transmission } \\
\text { energy\% }\end{array}$ \\
\hline Extremely high & $1-3$ & 1 & $1-3$ \\
Very high & $4-7$ & 1 & $4-7$ \\
High & $8-15$ & 1 & $8-15$ \\
Moderate & $16-21$ & 1 & $16-21$ \\
Low & $22-26$ & 1 & $21-26$ \\
Very low & $27-35$ & 1 & $27-35$ \\
\hline
\end{tabular}

Algorithm two: this algorithm is the opposite of the first algorithm as it applies the Shannon source coding theorem [44] for encoding. The information with the highest entropy is assigned with the shortest codeword, while the information with the lowest entropy is assigned the longest codeword. This algorithm can be applied in situations where the information gathering is intended for long term statistical analysis. While the Shannon target was to compress the information, the target here is to reduce energy consumption.

\section{RF-EH APPLICATIONS AND OPPORTUNITIES}

RF energy harvesting presents a real potential to many fields such as health monitoring, quality control disaster monitoring[32]. The values RF-EH offers are sustainability and cost efficiency by realizing power neutral wireless nodes. This section elaborates on some of the RF-EH applications and uses cases. Furthermore, some of the opportunities that $5 \mathrm{G}$ presents to the RF-EH field are discussed.

\subsection{Animal Health Tracking}

RF-EH sensors are used to track animal locations and collect information about their health status. Information collected through WSN may consist of the health status of the animals, habitat conditions, where 
the animals like to spend their time, and so forth. This information comes in handy when managing a large number of animals, for instance, a zoo or wildlife reserve. In [45], a multitier RF-EH WSN for animal healthcare is presented, as shown in Figure 13. In the first Tier, the biosensors mounted on the animal body send the collected data to a central node which is also mounted on the animal's body, forming a personal area network (BAN). In the second Tier, each central BAN node sends the gathered data to the nears Access point (AP), forming a local area network. In the third Tier, all the AP send their data to a central ethernet, usually placed in a health facility. As mentioned earlier, the main challenge of any RF-EH system is spreading loss. Thus, the energy efficiency of the sensor nodes and the placement of the HBS are crucial factors for such implementation.

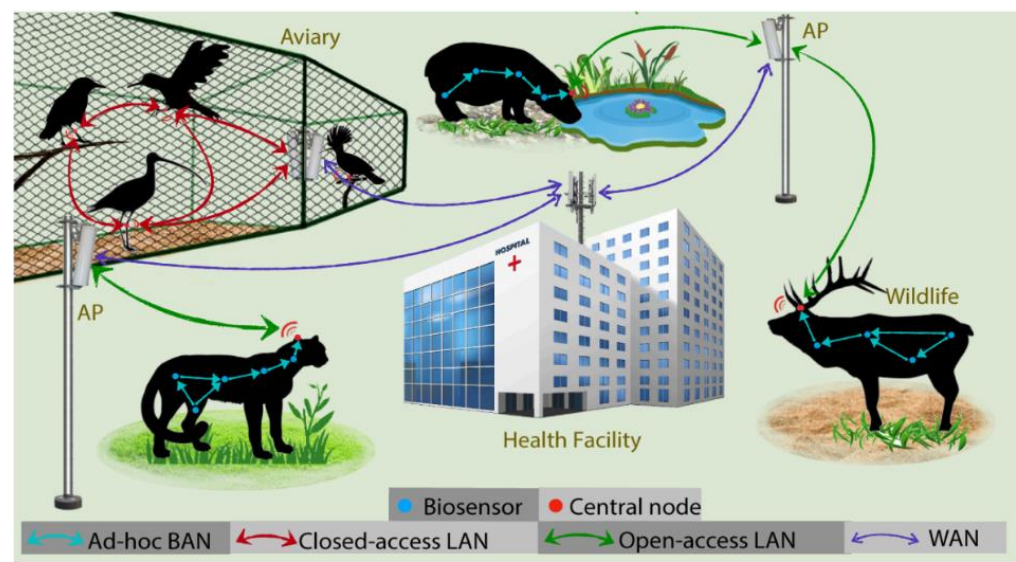

Figure 13. A three-tier Structure of RF-EHSNs for animal healthcare [45]

\subsection{Wearables}

Wearable devices such as smart bands and smartwatches have become wildly prevalent; nowadays, almost any smartphone manufacturer has its smart wearables line. These devices collect health meters like heartbeat and oxygen levels. Furthermore, it helps people to design body exercise plans based on the collected health meters. Currently, such devices exist as stand-alone system with a processing unit, large battery, data collection unit and display unit. If the data collection unit only is kept on the wristband and another device; for example, a smartphone is used for the remaining tasks, the band can be supported with RF energy as it requires less energy. In [46], an empirical experiment was carried out using a commercial two-way radio (walkie-talkie) as an energy transmitter to power a wearable device. The source transmission power was $2 \mathrm{w}$ with a frequency of $464-468 \mathrm{M} \mathrm{Hz}$. The highest received power was $6.9 \mathrm{dBm}$ when the power transmitter is placed in front of the wearable device, while the lowest received power was $-12 \mathrm{dBm}$ when the transmitter is placed behind the device. The distance between the transmitter and receiver varied between $0.5-1 \mathrm{~m}$. An implementation of PAN of wearables are presented in [47].

\subsection{G RF-EH opportunities}

Knowing the restrictions on the maximum transmittable power and high free space loss attenuation in RF-EH, 5G technologies may increase the possibility of harvesting more energy by improving the RF power density. Higher frequencies will have smaller wavelengths, enabling small-sized antennas that can be stacked together, as shown in Figure 14. The small antenna size and mm-wave penetrability issues will lead to the densification of the network. Thus, the distance between the EH node and the HBS will reduce significantly, leading to lower propagation losses. The increase in the number of antenna array elements will increase the gain at both the transmitter and receiver side; this can be achieved using spatial multiplexing techniques [48]. The gain is maximized when both the transmitter and receiver have an antenna array.

Using mm waves, the antenna array can form a high-resolution steerable beam. For instance, in [49], a phased array antenna with 32 elements is demonstrated with a beam steering flexibility of just 1.4 degrees between adjacent beams. Such beam steering will allow HBS to transmit to multiple firmly placed nodes at the same time.

\section{CONCLUSION}

RF-EH harvesting presents a real value in powering self-sustaining wireless networks by eliminating the need for conventional energy supply methods. This article presented a survey for the RF-EH hardware design, performance matrices. In addition, The information switching modes TS and PS are discussed. Furthermore, Power management in EH-SN is presented. Some of the current challenges like the limited RF 
power are discussed, and two algorithms are proposed passed on information criticality and the mode of the collected data. Finally, some of the RF-EH applications are elaborated, and the opportunities 5G offers to the RF-EH field are presented.

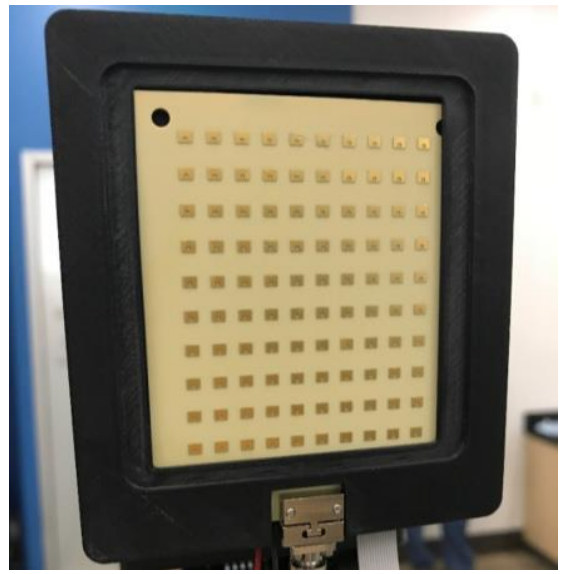

Figure14. A $28 \mathrm{GHz}$ Bi-directional face array antenna [50]

\section{ACKNOWLEDGEMENT}

We are very grateful to the Ministry of Higher education Malaysia for supporting this research under grant ID FRGS19-055-0663.

\section{REFERENCES}

[1] A. Israr, Q. Yang, W. Li, and A. Y. Zomaya, "Renewable energy powered sustainable 5G network infrastructure: Opportunities, challenges and perspectives," J. Netw. Comput. Appl., vol. 175, p. 102910, 2021.

[2] S. Shen, Y. Zhang, C.-Y. Chiu, and R. Murch, "An Ambient RF Energy Harvesting System Where the Number of Antenna Ports is Dependent on Frequency," IEEE Trans. Microw. Theory Tech., vol. 67, no. 9, pp. 3821-3832, 2019.

[3] Q. Li, H. Liu, H. Ning, Y. Fu, S. Hu, and S. Yang, "Supply and Demand Oriented Energy Management in the Internet of Things," Adv. Internet Things, vol. 06, no. 01, pp. 1-17, 2016.

[4] M. Cansiz, D. Altinel, and G. K. Kurt, "Efficiency in RF energy harvesting systems: A comprehensive review," Energy, vol. 174, pp. 292-309, 2019.

[5] H. S. Vu, N. Nguyen, N. Ha-Van, C. Seo, and M. Thuy Le, "Multiband Ambient RF Energy Harvesting for Autonomous IoT Devices," IEEE Microw. Wirel. Components Lett., vol. 30, no. 12, pp. 1189-1192, 2020

[6] G. Yang, Q. Zhang, and Y. C. Liang, "Cooperative ambient backscatter communications for green internet-ofthings," arXiv, vol. 5, no. 2, pp. 1116-1130, 2018.

[7] Y. Chen, Energy Harvesting Communications: Principles and Theories. 2019.

[8] A. Viswanathan, N. B. Sai Shibu, S. N. Rao, and M. V. Ramesh, "Security Challenges in the Integration of IoT with WSN for Smart Grid Applications," 2017 IEEE Int. Conf. Comput. Intell. Comput. Res. ICCIC 2017, pp. 03, 2018.

[9] P. K. Sharma, Y. S. Jeong, and J. H. Park, "EH-HL: Effective communication model by integrated EH-WSN and hybrid LiFi/WiFi for IoT," IEEE Internet Things J., vol. 5, no. 3, pp. 1719-1726, 2018.

[10] C. M. Yu, M. Tala'T, C. H. Chiu, and C. Y. Huang, "Joint balanced routing and energy harvesting strategy for maximizing network lifetime in WSNs," Energies, vol. 12, no. 12, pp. 1-20, 2019.

[11] G. Zhang, Y. Chen, Z. Shen, and L. Wang, "Distributed Energy Management for Multiuser Mobile-Edge Computing Systems With Energy Harvesting Devices and QoS Constraints," IEEE Internet Things J., vol. 6, no. 3, pp. 4035-4048, Jun. 2019.

[12] Y. Zhu, "Encoding Scheme to Reduce Energy Consumption of Delivering Data in Radio Frequency Powered Battery-Free Wireless Sensor Networks," vol. 67, no. 4, pp. 3085-3097, 2018.

[13] K. Tutuncuoglu and A. Yener, "Cooperative energy harvesting communications with relaying and energy sharing," 2013 IEEE Inf. Theory Work. ITW 2013, 2013.

[14] L. R. Varshney, "Transporting information and energy simultaneously," IEEE Int. Symp. Inf. Theory - Proc., pp. 1612-1616, 2008.

[15] P. Grover and A. Sahai, "Shannon meets Tesla: Wireless information and power transfer," pp. 2363-2367, 2010.

[16] A. Design, R. Tradeoff, X. Zhou, R. Zhang, and C. K. Ho, "Wireless Information and Power Transfer :," vol. 61, no. 11 , pp. 4754-4767, 2013.

[17] K. Singh, A. Gupta, T. Ratnarajah, and M. L. Ku, "A General Approach Toward Green Resource Allocation in Relay-Assisted Multiuser Communication Networks," IEEE Trans. Wirel. Commun., vol. 17, no. 2, pp. 848-862, 2018.

IJEEI, Vol.9, No. 1, March 2021: 101 - 113 
[18] Y. F. Su, R. R. Kotian, and N. Lu, "Energy harvesting potential of bendable concrete using polymer based piezoelectric generator," Compos. Part B Eng., vol. 153, pp. 124-129, Nov. 2018.

[19] C. A. Gueymard, "A reevaluation of the solar constant based on a 42-year total solar irradiance time series and a reconciliation of spaceborne observations," Sol. Energy, vol. 168, no. April, pp. 2-9, 2018.

[20] J. G. Kim, D. H. Kim, W. S. Yoo, J. Y. Lee, and Y. B. Kim, "Daily prediction of solar power generation based on weather forecast information in Korea," IET Renew. Power Gener., vol. 11, no. 10, pp. 1268-1273, 2017.

[21] W. Charge, "Energy Transmitter," 2019. [Online]. Available: https://wi-charge.com/product_category/referenceintegrations/.

[22] C. Julian Chen, Physics of Solar Energy. John Wiley \& Sons Inc, 2011.

[23] https://www.hydropower.org/, "Malaysia statistics," 2017. [Online]. Available: https://www.hydropower.org/country-profiles/malaysia. [Accessed: 02-Dec-2019].

[24] S. Priya et al., "A Review on Piezoelectric Energy Harvesting: Materials, Methods, and Circuits," Energy Harvest. Syst., vol. 4, no. 1, pp. 3-39, 2017.

[25] J. O. Ugwuogo, "On-Demand Energy Harvesting Techniques - A System Level Perspective," 2012.

[26] J. Bito, J. G. Hester, and M. M. Tentzeris, "Ambient RF Energy Harvesting from a Two-Way Talk Radio for Flexible Wearable Wireless Sensor Devices Utilizing Inkjet Printing Technologies," IEEE Trans. Microw. Theory Tech., vol. 63, no. 12, pp. 4533-4543, 2015.

[27] H. Stockman, "Communication by Means of Reflected Power," Proc. IRE, vol. 36, no. 10, pp. 1196-1204, 1948.

[28] A. R. Koelle, S. W. Depp, and R. W. FReyman, "Short-Range Radio-Telemetry for Electronic Identification, Using Modulated RF Backscatter," Proc. IEEE, vol. 63, no. 8, pp. 1260-1261, 1975.

[29] P. Nintanavongsa, U. Muncuk, D. R. Lewis, and K. R. Chowdhury, "Design optimization and implementation for RF energy harvesting circuits," IEEE J. Emerg. Sel. Top. Circuits Syst., vol. 2, no. 1, pp. 24-33, 2012.

[30] H. W. Pflug and H. J. Visser, Wireless Power Transfer Algorithms, Technologies and Applications in Ad Hoc Communication Networks. Cham: Springer International Publishing, 2016.

[31] K. W. Choi et al., "Simultaneous Wireless Information and Power Transfer (SWIPT) for Internet of Things: Novel Receiver Design and Experimental Validation," IEEE Internet Things J., vol. 7, no. 4, pp. 2996-3012, 2020.

[32] S. Selvan, M. Zaman, R. Gobbi, and H. Y. Wong, "Recent advances in the design and development of radio frequency-based energy harvester for powering wireless sensors: a review," J. Electromagn. Waves Appl., vol. 32, no. 16 , pp. 2110-2134, 2018.

[33] Skyworks Solutions, "Surface Mount Mixer and Detector Schottky Diodes," Datasheet, pp. 1-9, 2018.

[34] T. Le, K. Mayaram, and T. Fiez, "Efficient far-field radio frequency energy harvesting for passively powered sensor networks," IEEE J. Solid-State Circuits, vol. 43, no. 5, pp. 1287-1302, 2008.

[35] S. Park, J. Yang, and J. Rivas-Davila, "A Hybrid Cockcroft-Walton/Dickson Multiplier for High Voltage Generation," IEEE Trans. Power Electron., vol. 35, no. 3, pp. 2714-2723, 2020.

[36] S. K. Divakaran, D. Das Krishna, and Nasimuddin, "RF energy harvesting systems: An overview and design issues," International Journal of RF and Microwave Computer-Aided Engineering, vol. 29, no. 1. John Wiley and Sons Inc., p. e21633, 01-Jan-2019.

[37] T. N. Nguyen, T. H. Q. Minh, P. T. Tran, and M. Vozňák, "Energy harvesting over rician fading channel: A performance analysis for half-duplex bidirectional sensor networks under hardware impairments," Sensors (Switzerland), vol. 18, no. 6, 2018.

[38] Y. Luo, L. Pu, G. Wang, and Y. Zhao, "RF energy harvesting wireless communications: Rf environment, device hardware and practical issues," Sensors (Switzerland), vol. 19, no. 13, 2019.

[39] J. Yang and S. Ulukus, "Optimal Packet Scheduling in an Energy Harvesting Communication System," IEEE Trans. Commun., vol. 60, no. 1, pp. 220-230, Jan. 2012.

[40] Y. Luo, L. Pu, Y. Zhao, G. Wang, and M. Song, "Optimal energy requesting strategy for RF-based energy harvesting wireless communications," Proc. - IEEE INFOCOM, pp. 1-9, 2017.

[41] N. Grace, "Media Contact: FCC' S NEXT 5G SPECTRUM AUCTION IS UNDERWAY," vol. 385, no. 202, p. 1974, 2019

[42] M. Piñuela, P. D. Mitcheson, and S. Lucyszyn, "Ambient RF energy harvesting in urban and semi-urban environments," IEEE Trans. Microw. Theory Tech., vol. 61, no. 7, pp. 2715-2726, 2013.

[43] F. C. Commission, "FCC-19-126A1.pdf," 2019.

[44] C. E. Shannon, "A Mathematical Theory of Communication," Bell Syst. Tech. J., vol. 27, no. 3, pp. 379-423, 1948.

[45] Y. Luo, L. Pu, and Y. Zhao, "RF energy harvesting sensor networks for healthcare of animals: Opportunities and challenges," arXiv. pp. 1-6, 28-Feb-2018.

[46] B. S. Kim et al., "Wireless.Energy.Harvesting," vol. 102, no. 11, 2014.

[47] O. A. Saraereh, A. Alsaraira, I. Khan, and B. J. Choi, "A Hybrid Energy Harvesting Design for On-Body Internetof-Things (IoT) Networks," Sensors, vol. 20, no. 2, p. 407, Jan. 2020.

[48] S. Buzzi, I. Chih-Lin, T. E. Klein, H. V. Poor, C. Yang, and A. Zappone, "A survey of energy-efficient techniques for 5G networks and challenges ahead," IEEE J. Sel. Areas Commun., vol. 34, no. 4, pp. 697-709, 2016.

[49] C. Dual and P. Beams, "To demonstrate the resulting output power vs NF trade-off, Fig. 7.2.3 compares the TX front-end (FE) OP," IEEE Int. Solid-state Circuits Conf., pp. 128-130, 2017.

[50] University of California, "Keysight Technologies, University of California San Diego Demonstrate the World's Fastest $28 \mathrm{GHz}$ 5G Band, Bidirectional Phased-Array," 2017. [Online]. Available: http://cwc.ucsd.edu/news/keysight-technologies-university-california-san-diego-demonstrate-worlds-fastest-28ghz-5g. 